\title{
A NOTE ON IDENTIFICATIONS OF METRIC SPACES
}

FRANK SIWIEC

\author{
Dedicated to Professor Jun-iti Nagata
}

\begin{abstract}
A space $X$ is said to be $\sigma M K$ provided that $X$ has a countable closed cover $C$ of metrizable subspaces such that if $K$ is a compact subset of $X$, there is a $C \in \mathcal{C}$ for which $K \subset C$. A Hausdorff space is $\sigma M K$ and Fréchet if and only if it is representable as a closed image of a metric space obtained by identifying a discrete collection of closed sets with hemicompact boundaries to points.
\end{abstract}

A familiar example of a nonmetrizable space is $R / N$, that is, the space obtained by identifying the set of natural numbers $N$, in the set of real numbers $R$, to a point and giving the resulting set the quotient topology. In [5], the concept of a $\sigma M K$ space proved useful in characterizing certain countably infinite spaces. This note relates identification spaces such as $R / N$ with the concept of a $\sigma M K$ space.

All spaces in this paper are understood to be Hausdorff topological spaces and all mappings are continuous onto functions. A space $X$ is $\sigma M K$ provided that $X$ has a countable closed cover $C$ of metrizable subspaces such that if $K$ is a compact subset of $X$, there is a $C \in \mathcal{C}$ for which $K \subset C$. We may assume that $C$ consists of sets $C_{1} \subset C_{2} \subset \cdots$, and we will henceforth do so. A space $X$ is Fréchet [2] provided that every accumulation point of a set $A$ in $X$ is the limit of some sequence in $A$. It is clear that $\sigma M K$ and Fréchet are each hereditary properties.

THEOREM 1. If a space $X$ is $\sigma M K$ and Fréchet, then it is an image of a metric space $M$ under a closed mapping $f$, and there is a discrete collection $\mathcal{F}$ of closed subsets of $M$, such that $f(F)$ is a point for each $F \in \mathscr{F}$, Bdy $F$ is hemicompact for each $F \in \mathscr{F}$, and $f$ is one-to-one upon restriction to $M-\cup \mathscr{F}$.

The proof follows from a number of propositions.

The concept of a $\sigma M K$ space arose in analogy to the concept of hemicompactness introduced by Arens [1]. A space $X$ is hemicompact provided that there is a countable cover $C$ of compact subspaces such that if $K$ is a compact subset of $X$, there is a $C \in \mathcal{e}$ for which $K \subset C$. A metrizable space is hemicompact if and only if it is separable and locally compact.

Proposition 2. (a) If a space $X$ is $\sigma M K$ and Fréchet, then it is a closed image of a metric space having cardinality that of $X$.

Presented to the Society, November 7, 1975; received by the editors September 26, 1974 and, in revised form, May 18, 1975.

AMS (MOS) subject classifications (1970). Primary 54E20; Secondary 54C10.

Key words and phrases. $\sigma M K$ space, closed image of a metric space. 
(b) A space $X$ is hemicompact, Fréchet, and has every compact subspace metrizable if and only if it is a closed image of a locally compact separable metric space. (The metric space may be chosen to have cardinality that of $X$.)

Proof. We need only prove case (a), since case (b) is similar. (The "if" of case (b) is well known. In part, see [3].) Thus, assume that $X$ is Fréchet and $X=\cup\left\{C_{n} \mid n \in N\right\}$ as given in the definition of $\sigma M K$.

Let $C_{0}=\varnothing$. Let $M_{n}=\operatorname{cl}\left(C_{n}-C_{n-1}\right)$ for each $n$. Let $M$ be the discrete union of the $M_{n}$ and let $f$ be the natural mapping of $M$ onto $X$. Clearly $M$ is metrizable and $f$ is continuous. We need to show that $f$ is closed. Let $x_{0}$ be a point of $X$ for which there is a sequence $\left\{x_{i}\right\}$ of distinct points of $X-\left\{x_{0}\right\}$ converging to $x_{0}$. It suffices to show that if points $p_{i} \in f^{-1}\left(x_{i}\right)$ are chosen for each $i$, then the sequence $\left\{p_{i}\right\}$ has a convergent subsequence in the space $M$. So, let $p_{i} \in f^{-1}\left(x_{i}\right)$ for each $i$.

We will need the fact that there exists an integer $n_{0}$ for which $\left\{x_{0}, x_{1}\right.$, $\left.x_{2}, \ldots\right\}$ is contained in $\bigcup\left\{M_{n} \mid n=1,2, \ldots, n_{0}\right\}$, and $x_{i} \notin M_{n}$ for $i$ $\in N$ and $n>n_{0}$. Suppose not. Then there exists a subsequence $\left\{x_{i}\right\}$ of $\left\{x_{i}\right\}$ and a subsequence $\left\{n_{j}\right\}$ of $N$ such that $x_{i_{j}} \in \operatorname{cl}\left(C_{n_{j}+1}-C_{n_{j}}\right)$ for each $j$, with $n_{j} \neq n_{k}$ for distinct $j, k$. Then for each $j$, there is a sequence $\left\{x_{k} \mid k \in N\right\}$ $\subset C_{n_{j}+1}-C_{n_{j}}$ such that $x_{k} \rightarrow x_{i_{j}}$. By the Fréchet assumption, since $x_{0}$ is an accumulation point of the set $\left\{x_{k}^{l} \mid k, l \in N\right\}$, there is a sequence $\left\{q_{j}\right\}$ contained in $\left\{x_{k}^{l} \mid k, l \in N\right\}$ such that $q_{j} \rightarrow x_{0}$ and $q_{j} \neq x_{0}$ for all $j$. Let $F$ be the set $\left\{q_{j} \mid j \in N\right\}$. Then for each $n, F \cap C_{n}$ is finite and so closed. Since $X$ is $\sigma M K$ and Fréchet, $F$ must be closed. This is impossible, so our supposition is false.

By the fact that we have just shown, there is a subsequence $\left\{x_{i_{i}}\right\}$ and there is an integer $n_{1} \leqslant n_{0}$ for which $\left\{x_{0}, x_{i_{1}}, x_{i_{2}}, \ldots\right\}$ is contained in $M_{n_{1}}$, and for each $n>n_{1}, x_{i_{j}} \in M_{n}$ for at most finitely many $j \in N$. Thus there is an $n_{2} \leqslant n_{1}$ for which a subsequence of $\left\{p_{1}, p_{2}, \ldots\right\}$ is contained in $M_{n_{2}}$. This subsequence of $\left\{p_{1}, p_{2}, \ldots\right\}$ converges in $\boldsymbol{M}_{\boldsymbol{n}_{2}}$, and so also in $\boldsymbol{M}$.

Lemma 3. Suppose $S=\{0, i,(i, j, k) \mid i, j, k \in N\}$ has a topology with the following properties. Each point $(i, j, k)$ is itself an open set. Each set $S_{i}$ $=\{i,(i, j, k) \mid j, k \in N\}$ is an open set and is homeomorphic to the "sequential fan" (that is, a set $G$ is a neighborhood of $i$ in $S_{i}$ if $i \in G$, and for each $j,(i, j, k) \in G$ for all but finitely many $k)$. The sequence $\{i\}$ converges to 0 . Then $S$ cannot be both $\sigma M K$ and Fréchet.

Proof. Suppose on the contrary that $S$ is Fréchet and $S=\cup\left\{C_{i} \mid i \in N\right\}$ as given by the definition of $\sigma M K$. We may assume (without loss of generality) that $\{0,1,2, \ldots\} \subset C_{1}$. Notice that for each $i, S_{i}$ is not contained in $C_{i}$, since $S_{i}$ is not metrizable. In fact, for each $i, S_{i}-C_{i}$ must contain a sequence $S_{i}^{\prime}=\left\{\left(i, j, k_{n}\right) \mid n \in N\right\}$ for some $j \in N$ and some subsequence $\left\{k_{n}\right\}$ of $\{k \mid k$ $\in N\}$. Let $S^{\prime}=\bigcup\left\{S_{i}^{\prime} \mid i \in N\right\}$. Then 0 is an accumulation point of $S^{\prime}$. Since $S$ is assumed to be Fréchet, there is a sequence $T$ in $S^{\prime}$ which converges to 0 . But then, there is an integer $i_{0}$ for which $T \subset C_{i_{0}}$. Since $T \subset S^{\prime}$ and $T$ converges to 0 , there is a point $x_{0}$ common to $T$ and $\bigcup\left\{S_{i}^{\prime} \mid i \geqslant i_{0}\right\}$. Let $i_{1} \geqslant i_{0}$ be such that $x_{0} \in T \cap S_{i_{1}}^{\prime}$. Then $x_{0} \in T \subset C_{i_{0}} \subset C_{i_{1}}$. Thus, $x_{0} \in S_{i_{1}}^{\prime}$ $\cap C_{i_{1}}$. This is a contradiction.

Proposition 4. If a space $X$ is $\sigma M K$ and Fréchet, and $D$ is the set of those 
points of $X$ at which $X$ is not first-countable, then no point of $X$ is an accumulation point of $D$.

Proof. Otherwise, there exists a sequence $\left\{x_{n}\right\}$ of distinct points converging to a point $x_{0} \in X$ such that each $x_{n}$ (for $n=1,2, \ldots$ ) is a point of non-firstcountability and $x_{n} \neq x_{0}$. There exists a sequence of disjoint open sets $G_{n}$ such that $x_{n} \in G_{n}$ for $n=1,2, \ldots$. Since each $G_{n}$ is Fréchet, but not countably bisequential at $x_{n}$ (since a closed image of a metric space which is countably bisequential is metrizable), by [6], there exists a copy of the sequential fan in $G_{n}$ "at $x_{n}$ ". Let $S_{n}$ denote this copy. Thus, for each $n=1,2, \ldots$, there is an $S_{n}$ "at $x_{n}$ " and these $S_{n}$ are disjoint. Let $S$ $=\cup\left\{S_{n} \mid n=1,2, \ldots\right\} \cup\left\{x_{0}\right\}$. By Lemma $3, S$ is either not $\sigma M K$ or not Fréchet. We have a contradiction.

Proposition 5. If a space $X=M / F$, where $X$ is $\sigma M K, M$ is metrizable, and $F$ is a closed subset of $M$, then Bdy $F$ is hemicompact.

Proof. If Bdy $F$ is empty, it is trivially hemicompact. If Bdy $F$ is nonempty, $M / F=(M-$ Int $F) /$ Bdy $F$, so we may assume (without loss of generality) that the interior of $F$ is empty. Let $X=\cup\left\{C_{n} \mid n \in N\right\}$ as given by the definition of $\sigma M K$. Let $f$ be the natural mapping of $M$ into $X$. Since there exists an $n \in N$ for which the point $f(F) \in C_{n}$, we may assume that in fact, $f(F) \in C_{1}$. Since $C_{n}$ is metrizable, $f_{n}=f \mid f^{-1}\left(C_{n}\right)$ is a closed mapping of $f^{-1}\left(C_{n}\right)$ onto $C_{n}$ with Bdy $f_{n}^{-1}(x)$ being compact for each point $x$ of $C_{n}$ ([4] or [7]). Let $K_{n}=$ Bdy $f_{n}^{-1}(f(F))$ for each $n$, where the boundary is taken relative to $f^{-1}\left(C_{n}\right)$. Then each $K_{n}$ is compact. Also, $F=\bigcup\left\{K_{n} \mid n \in N\right\}$. Because, if $p \in F$, there is a sequence $\left\{p_{i}\right\}$ in $M-F$ which converges to $p$. But there is an integer $n_{0}$ for which $\left\{f(p), f\left(p_{1}\right), f\left(p_{2}\right), \ldots\right\} \subset C_{n_{0}}$. Thus, $p$ $\in \operatorname{cl}\left(f^{-1}\left(C_{n_{0}}\right)-F\right) \cap F=K_{n_{0}}$.

In order to show that $\left\{K_{n} \mid n \in N\right\}$ is a sequence as in the definition of hemicompact, let $K$ be a compact subset of $F$. Suppose that for all $n$, there is a point $x_{n} \in K-K_{n} \subset K-\mathrm{cl}\left(f^{-1}\left(C_{n}\right)-F\right)$. Since $K$ is sequentially compact, there is a point $x$ in $K$ which is the limit of some subsequence of $\left\{x_{n}\right\}$. For simplicity of notation, assume that the subsequence is $\left\{x_{n}\right\}$ itself. Let $\left\{G_{n} \mid n \in N\right\}$ be an open base at $x$ in $M$ such that $G_{n} \supset G_{n+1}$ for all $n$. We may also assume that $x_{n} \in G_{n}$ for all $n$. Since $x_{n}$ is in (the boundary of) $F$, there exists a sequence $\left\{x_{m}^{n} \mid m \in N\right\}$ in $G_{n}-F$ which converges to $x_{n}$. Let $L$ $=\left\{x, x_{n}, x_{m}^{n} \mid m, n \in N\right\}$. Then $L$ is a compact subset of $M$. Since $f(L)$ is compact in $X$, there is an integer $n_{0}$ for which $f(L) \subset C_{n_{0}}$. Then the sequence $\left\{x_{m}^{n_{0}} \mid m \in N\right\}$ is contained in $f^{-1}\left(C_{n_{0}}\right)-F$. This means that $x_{n_{0}}$ $\in \mathrm{cl}\left(f^{-1}\left(C_{n_{0}}\right)-F\right)$. We have a contradiction, and thereby we have shown that (the boundary of) $F$ is hemicompact.

Proof of Theorem 1. Let $X$ be $\sigma M K$ and Fréchet. By Proposition 2, $X$ is a closed image of a metric space $M^{\prime}$ under a mapping $h$. Let $D$ be the set of those points of $X$ at which $X$ is not first-countable. By Proposition 4, $D$ is a discrete closed subspace of $X$, and ([4] or [7]) $X-D$ is metrizable. Let $M=h^{-1}(D) \cup(X-D)$, define $g: M^{\prime} \rightarrow M$ by $g \mid h^{-1}(D)=$ identity, $g\left|h^{-1}(X-D)=h\right| h^{-1}(X-D)$, and $f: M \rightarrow X$ by $f\left|h^{-1}(D)=h\right| h^{-1}(D)$, $f \mid(X-D)=$ identity. Giving $M$ the quotient topology as an image of $M^{\prime}, g$ 
and $f$ are closed continuous mappings and $M$ is metrizable. Let $\mathscr{F}=\left\{f^{-1}(x) \mid\right.$ $x \in D\}$. Then $\mathscr{F}$ is a discrete collection in $M$ and by Proposition 5, each $F \in \mathscr{F}$ has a hemicompact boundary. The proof is complete.

We now prove the converse of Theorem 1 .

THEOREM 6. If a space $X$ is an image of a metric space $M$ under a closed mapping $f$, and there is a discrete collection $\mathscr{F}$ of closed subsets of $M$, such that $f(F)$ is a point for each $F \in \mathscr{F}$, Bdy $F$ is hemicompact for each $F \in \mathscr{F}$, and $f$ is one-to-one upon restriction to $M-\cup \mathscr{F}$, then $X$ is $\sigma M K$ and Fréchet.

Proof. It is well known that a closed image of a metric space is Fréchet. We prove that $X$ is $\sigma M K$ under the stated hypotheses. We may assume (without loss of generality) that Int $F=\varnothing$ for each $F \in \mathscr{F}$. Let each $F=\cup\left\{C_{n}^{F} \mid n \in N\right\}$ as given by the definition of hemicompact, and we may assume that each $C_{n}^{F} \subset C_{n+1}^{F}$. Since $\mathcal{F}$ is a discrete collection in $M$, there exists a discrete collection $\left\{G^{F} \mid F \in \mathscr{F}\right\}$ of open sets such that $F \subset G^{F}$ for every $F \in \mathscr{F}$. Also, let $G_{n}^{F}=G^{F} \cap S_{1 / n}\left(F-C_{n}^{F}\right)$, where $S_{1 / n}(A)$ denotes the $1 / n$ open sphere around set $A$. Let $D_{n}^{F}=C_{n}^{F}-G_{n}^{F}$. Finally, let $M_{n}^{\prime}=M$ - $\cup\left\{G_{n}^{F} \mid F \in \mathscr{F}\right\}, f_{n}=f \mid M_{n}^{\prime}$, and $M_{n}=f_{n}\left(M_{n}^{\prime}\right)$ for all $n$. Since $f$ is a closed mapping and $M_{n}^{\prime}$ is a closed subset of $M$, each $f_{n}$ is a closed mapping. Also, $f_{n}^{-1}(x)$ is compact for each point $x$ of $X$, since each $D_{n}^{F}$ is compact. By [4] or [7], each $M_{n}$ is then metrizable. It is clear that $X=\cup\left\{M_{n} \mid n \in N\right\}$.

Now let $K$ be a compact subset of $X$. And suppose that $K \nsubseteq M_{n}$ for all $n$. Then for each $n$, there is a point $x_{n} \in K-M_{n}$. But $K$ is sequentially compact (being compact and Fréchet), and so there is a subsequence $\left\{x_{n_{i}}\right\}$ of distinct points, converging to a point $x$ of $K$, with $x_{n_{i}} \neq x$ for all $i$. Fix $m \in N$. Since the sequence $\left\{x_{n_{i}}\right\}$ meets $M_{m}$ in at most finitely many points, there exists an $i_{m} \in N$ such that $x_{n_{i}} \in f\left(\cup\left\{G_{m}^{F} \mid F \in \mathscr{F}\right\}\right)$ for all $i>i_{m}$. For each $i>i_{m}$, let $y_{n_{i}} \in f^{-1}\left(x_{n_{i}}\right) \cap \cup\left\{G_{m}^{F} \mid F \in \mathscr{F}\right\}$. Since the set $\left\{y_{n_{i}} \mid i>i_{m}\right\}$ is not closed in $M$, let $y$ be an accumulation point of this set. So there exists a subsequence of $\left\{y_{n_{i}}\right\}$ which converges to $y$. This means that $f(y)=x$. Also, $y \in \operatorname{cl} \cup\left\{G_{m}^{F} \mid F\right.$ $\in \mathscr{F}\} \subset \cup\left\{G_{m-1}^{F} \mid F \in \mathscr{F}\right\}$ for all $m$. We then have that $y \in F_{0}$ for some $F_{0} \in \mathscr{F}_{\text {. Since }} y \in G_{m}^{F_{0}} \subset S_{1 / m}\left(F_{0}-C_{m}^{F_{0}}\right)$ for every $m$, let $p_{m}$ be a point of $F_{0}-C_{m}^{F_{0}}$ for which $d\left(y, p_{m}\right)<1 / m$. Then $p_{m} \rightarrow y$ in $F_{0}$. So there is an integer $n_{0}$ for which $\left\{y, p_{1}, p_{2}, \ldots\right\} \subset C_{n_{0}}^{F_{0}}$. Thus $p_{n_{0}} \in C_{n_{0}}^{F_{0}}$. This is a contradiction.

EXAMPLES 7. To illustrate the results of this paper we consider two examples of countable regular Fréchet spaces. Let $Q$ denote the usual space of rational numbers. Let $Q^{\prime}$ be $Q^{2}$ in the plane together with the entire $x$-axis of real numbers, with the usual topology from the plane. Let $X_{1}$ be the quotient space obtained by considering $Q^{\prime}$ and identifying the $x$-axis to a point. Let $X_{2}$ be the quotient space obtained by considering $Q^{2}$ and identifying the set $Q$ in the $x$ axis to a point. Then $X_{1}$ and $X_{2}$ are the desired spaces. By Theorem $6, X_{1}$ is $\sigma M K$. On the other hand, $X_{2}$ is not $\sigma M K$. To see this, suppose $X_{2}$ $=\bigcup\left\{C_{n} \mid n \in N\right\}$ as given by the definition of $\sigma M K$ and suppose that $C_{n} \subset C_{n+1}$ for all $n$. Since $X_{2}$ is not metrizable, for each $n$ there exists a point $x_{n}$ in $\left[\left([0,1 / n]^{2} \cap Q^{2}\right) / Q\right]-C_{n}$. Then $x_{n} \rightarrow(0,0)$. So there exists an integer $n_{0}$ for which $\left\{x_{n} \mid n \in N\right\} \subset C_{n_{0}}$, contradiction. 


\section{REFERENCES}

1. R. F. Arens, A topology for spaces of transformations, Ann. of Math. (2) 47 (1946), $480-495$. MR 8, 165.

2. S. P. Franklin, Spaces in which sequences suffice, Fund. Math. 57 (1965), 107-115. MR 31 \#5184.

3. K. Morita, On decomposition spaces of locally compact spaces, Proc. Japan Acad. 32 (1956), 544-548. MR 19, 49.

4. K. Morita and S.Hanai, Closed mappings and metric spaces, Proc. Japan Acad. 32 (1956), 10-14. MR 19, 299.

5. F. Siwiec, Countable spaces having exactly one nonisolated point. I, Proc. Amer. Math. Soc. 57 (1976), 345-350.

6. - Generalizations of the first axiom of countability, Rocky Mountain J. Math. 5 (1975), $1-60$.

7. A. H. Stone, Metrizability of decomposition spaces, Proc. Amer. Math. Soc. 7 (1956), 690-700. MR 19, 299.

Department of Mathematics, John Jay College of the City University of New York, New YorK, New YoRK 10019 